\title{
The coupling integrable couplings of the generalized coupled Burgers equation hierarchy and its Hamiltonian structure
}

\author{
Yuan Wei ${ }^{1 *}$, Li Yin ${ }^{1}$ and Xin Long ${ }^{2,3}$
}

"Correspondence:

weiyuan_1979@163.com

${ }^{1}$ College of Science, Binzhou

University, Binzhou City, P.R. China

Full list of author information is

available at the end of the article

\begin{abstract}
In this paper, we mainly give the Lie algebras $E, F$ and $H$ of three kinds and their commutator, respectively. Next, we establish three isospectral problems with the help of their corresponding loop algebras $\widetilde{E}, \widetilde{F}$, and $\widetilde{H}$. Based on on the Tu scheme, coupling integrable couplings of three kinds for the generalized coupled Burgers equation hierarchy are obtained. Finally, we obtain the Hamiltonian structure of one of the coupling integrable couplings of the generalized coupled Burgers equation hierarchy by using the quadratic-form identity.
\end{abstract}

MSC: 35B06

Keywords: Isospectral problem; Burgers equation hierarchy; Quadratic-form identity; Hamiltonian structure

\section{Introduction}

The development of soliton theory has undergone a rapid development in the 1960s. Integrable couplings are a new important and interesting topic in the field of soliton theory [13]. A lot of integrable nonlinear evolution equations, such as the Schroedinger equation and the $\mathrm{KdV}$ equation, were discussed. The notion of integrable couplings was first introduced by Virasoro [4, 5]. Integrable couplings are coupled systems of integrable equations which contain given integrable equations as their sub-systems [6]. Integrable couplings have much richer mathematical structures than scalar integrable equations [7-31]. Recently, Inc, Yusuf, Aliyu and Baleanu discussed a Lie symmetry analysis and conservation laws for the time fractional simplified modified Kawahara equation, the generalized shallow water wave equation, the time fractional dispersive long-wave equation and the time fractional generalized Burgers-Huxley equation. They also studied a time fractional thirdorder variant Boussinesq system and gave a symmetry analysis, explicit solutions, conservation laws and numerical approximations [32-37]. So it is important to study integrable couplings in soliton theory. Zhang even proposed an efficient method for constructing nonlinear evolution equations and their resulting Hamiltonian structure. Ma called it the Tu scheme. Hence, Hu developed the trace identity, and got an efficient method to work out the soliton equations and the Hamiltonian structure. In [38] Zhang gave three kinds of coupling integrable couplings of the KdV hierarchy of evolution equations. The three Lie algebras $E, F$ and $H$ in [38] can be used to obtain the other coupling integrable couplings

(c) The Author(s) 2019. This article is distributed under the terms of the Creative Commons Attribution 4.0 International License (http://creativecommons.org/licenses/by/4.0/), which permits unrestricted use, distribution, and reproduction in any medium, provided you give appropriate credit to the original author(s) and the source, provide a link to the Creative Commons license, and indicate if changes were made. 
of the soliton equations. In this paper, we introduce three higher-dimensional Lie algebras and their corresponding loop algebras $\widetilde{E}, \widetilde{F}$, and $\widetilde{H}$, and consider three Lax pairs for the zero curvature equation

$$
V_{x}=[U, V] .
$$

With the help of the loop algebras, we obtain three kinds of coupling integrable couplings of the generalized coupled Burgers equation hierarchy. And the one coupling integrable coupling of the generalized coupled Burgers equation hierarchy has a Hamiltonian structure obtained by employing the quadratic-form identity [39].

\section{Three higher-dimensional Lie algebras}

Let

$$
E=\operatorname{span}\left\{e_{1}, e_{2}, e_{3}, e_{4}, e_{5}, e_{6}, e_{7}\right\}
$$

For $\forall a=\sum_{i=1}^{7} a_{i} e_{i}, b=\sum_{i=1}^{7} b_{i} e_{i} \in E$, we have

$$
[a, b]=\left(\begin{array}{c}
2 a_{3} b_{2}-2 a_{2} b_{3} \\
2 a_{1} b_{3}-2 a_{3} b_{1} \\
2 a_{1} b_{2}-2 a_{2} b_{1} \\
a_{1} b_{4}-a_{4} b_{1}+a_{2} b_{5}-a_{5} b_{2}+a_{3} b_{5}-a_{5} b_{3} \\
a_{5} b_{1}-a_{1} b_{5}+a_{2} b_{4}-a_{4} b_{2}+a_{4} b_{3}-a_{3} b_{4} \\
a_{1} b_{6}-a_{6} b_{1}+a_{2} b_{7}-a_{7} b_{2}+a_{3} b_{7}-a_{7} b_{3} \\
a_{7} b_{1}-a_{7} b_{1}+a_{2} b_{6}-a_{6} b_{2}+a_{6} b_{3}-a_{3} b_{6}
\end{array}\right) .
$$

It is easy to see that $E$ is a Lie algebra equipped with the commutator (2.1).

Denote

$$
F=\operatorname{span}\left\{f_{1}, f_{2}, f_{3}, f_{4}, f_{5}, f_{6}, f_{7}, f_{8}, f_{9}\right\} .
$$

For $\forall a=\sum_{i=1}^{7} a_{i} f_{i}, b=\sum_{i=1}^{7} b_{i} f_{i} \in L_{2}$, we easily obtain

$$
[a, b]=\left(\begin{array}{c}
2 a_{3} b_{2}-2 a_{2} b_{3} \\
2 a_{1} b_{3}-2 a_{3} b_{1} \\
2 a_{1} b_{2}-2 a_{2} b_{1} \\
2 a_{7} b_{2}-2 a_{2} b_{7}+2 a_{3} b_{6}-2 a_{6} b_{3} \\
2 a_{3} b_{8}-2 a_{8} b_{3}+2 a_{9} b_{2}-2 a_{2} b_{9} \\
2 a_{1} b_{7}-2 a_{7} b_{1}+2 a_{4} b_{3}-2 a_{3} b_{4} \\
2 a_{1} b_{6}-2 a_{6} b_{1}+2 a_{4} b_{2}-2 a_{2} b_{4} \\
2 a_{1} b_{9}-2 a_{9} b_{1}+2 a_{5} b_{3}-2 a_{3} b_{5} \\
2 a_{1} b_{8}-2 a_{8} b_{1}+2 a_{5} b_{2}-2 a_{2} b_{5}
\end{array}\right)
$$

and $F$ is a Lie algebra equipped with the commutator (2.2).

The Lie algebras $E$ and $F$ are all expanding Lie algebra of the well-known Lie algebra

$$
R^{3}=\left\{r=\left(r_{1}, r_{2}, r_{3}\right)^{T}, r_{i} \in R\right\},
$$


which along with the commutator leads to

$$
[a, b]=\left(\begin{array}{c}
2 a_{3} b_{2}-2 a_{2} b_{3} \\
2 a_{1} b_{3}-2 a_{3} b_{1} \\
2 a_{1} b_{2}-2 a_{2} b_{1}
\end{array}\right) \quad \forall a=\left(a_{1}, a_{2}, a_{3}\right)^{T}, b=\left(b_{1}, b_{2}, b_{3}\right)^{T} \in R^{3}
$$

Assume that $L_{s}$ is a $s$-dim Lie algebra,

$$
K\left(L_{s}\right)=\left\{A=\left(\begin{array}{l}
A_{1} \\
A_{2} \\
A_{3}
\end{array}\right), A_{i} \in L_{s}, i=1,2,3\right\} .
$$

For

$$
B=\left(\begin{array}{l}
B_{1} \\
B_{2} \\
B_{3}
\end{array}\right) \in K\left(L_{s}\right)
$$

and

$$
[A, B]=\left(\left[A_{1}, B_{1}\right],\left[A_{1}, B_{2}\right]+\left[A_{2}, B_{1}\right],\left[A_{1}, B_{3}\right]+\left[A_{3}, B_{1}\right]+\left[A_{3}+B_{3}\right]\right)^{T} \in K\left(L_{s}\right),
$$

$K\left(L_{s}\right)$ is a Lie algebra. By taking $s=3$ and $\left[A_{1}, B_{1}\right]$ is the same as (2.3), then we can get another Lie algebra $K\left(L_{s}\right)$, which can be denoted $H$. The Lie algebra $H$ is isomorphic to the Lie algebra $F$,

$$
H=\operatorname{span}\left\{f_{1}, f_{2}, f_{3}, f_{4}, f_{5}, f_{6}, f_{7}, f_{8}, f_{9}\right\}
$$

along with the commutator

$$
[a, b]=\left(\begin{array}{c}
2 a_{3} b_{2}-2 a_{2} b_{3} \\
2 a_{1} b_{3}-2 a_{3} b_{1} \\
2 a_{1} b_{2}-2 a_{2} b_{1} \\
2 a_{3} b_{5}-2 a_{5} b_{3}+2 a_{6} b_{2}-2 a_{2} b_{6} \\
2 a_{1} b_{6}-2 a_{6} b_{1}+2 a_{4} b_{3}-2 a_{3} b_{4} \\
2 a_{1} b_{5}-2 a_{5} b_{1}+2 a_{4} b_{2}-2 a_{2} b_{4} \\
2 a_{3} b_{8}-2 a_{8} b_{3}+2 a_{9} b_{2}-2 a_{2} b_{9}+2 a_{9} b_{8}-2 a_{8} b_{9} \\
2 a_{1} b_{9}-2 a_{9} b_{1}+2 a_{7} b_{3}-2 a_{3} b_{7}+2 a_{7} b_{9}-2 a_{9} b_{7} \\
2 a_{1} b_{8}-2 a_{8} b_{1}+2 a_{7} b_{2}-2 a_{2} b_{7}+2 a_{7} b_{8}-2 a_{8} b_{7}
\end{array}\right),
$$

where $a=\sum_{i=1}^{9} a_{i} e_{i}, b=\sum_{i=1}^{9} b_{i} e_{i}$.

By applying Lie algebra $E, F$ and $H$, we can obtain three kinds of coupling integrable couplings of the generalized coupled Burgers equation hierarchy.

\section{Three kinds of coupling integrable couplings of the generalized coupled Burgers equation hierarchy}

In this section, we shall show how to get three kinds of coupling integrable couplings of the generalized coupled Burgers equation hierarchy by making use of the Lie algebra $E, F$ and $H$. 
(I) The first kind of coupling integrable couplings of the generalized coupled Burgers equation hierarchy.

The loop algebra of Lie algebra $E$ is given by $\widetilde{E}=\operatorname{span}\left\{e_{i}(n)=e_{i} \lambda^{n}, i=1,2,3,4,5,6,7\right\}$, $\left[e_{i}(m), e_{j}(n)\right]=\left[e_{i}, e_{j}\right] \lambda^{m+n}, m, n \in Z, i, j=1,2,3,4,5,6,7$, equipped with the commutator (2.1). Consider a Lax pair for zero curvature equation as follows:

$$
\left\{\begin{aligned}
U= & e_{1}(1)+q h_{2}(0)+h_{2}(1)+r h_{3}(0)+u_{1} h_{4}(0)+u_{2} h_{5}(0)+s_{1} h_{6}(0)+s_{2} h_{7}(0), \\
V= & \sum_{m \geq 0}\left(V_{1 m} h_{1}(-m)+V_{2 m} h_{2}(-m)+V_{3 m} h_{3}(-m)+V_{4 m} h_{4}(-m)\right. \\
& \left.+V_{5 m} h_{5}(-m)+V_{6 m} h_{6}(-m)+V_{7 m} h_{7}(-m)\right) .
\end{aligned}\right.
$$

The stationary zero equation

$$
V_{x}=[U, V]
$$

changes into

$$
\left\{\begin{array}{l}
V_{1, m x}=-2 V_{3, m+1}-2 q V_{3 m}+2 r V_{2 m}, \\
V_{2, m x}=2 V_{3 m}-2 r V_{1 m}, \\
V_{3, m x}=-2 V_{1, m+1}-2 q V_{1 m}+2 V_{2 m} \\
V_{4, m x}=V_{5, m+1}+q V_{5 m}+V_{4 m}-u_{1} V_{1 m}-u_{2} V_{2 m}+r V_{5 m}-u_{2} V_{3 m} \\
V_{5, m x}=V_{4, m+1}+q V_{4 m}+u_{2} V_{1 m}-V_{5 m}-u_{1} V_{2 m}+u_{1} V_{3 m}-r V_{4 m} \\
V_{6, m x}=V_{7, m+1}+q V_{7 m}+V_{6 m}-s_{1} V_{1 m}-s_{2} V_{2 m}+r V_{7 m}-s_{2} V_{3 m} \\
V_{7, m x}=V_{6, m+1}+q V_{6 m}+s_{2} V_{1 m}-V_{7 m}-s_{1} V_{2 m}+s_{1} V_{3 m}-r V_{6 m} .
\end{array}\right.
$$

Denoting

$$
\begin{aligned}
V_{+}^{(n)}= & \sum_{m=0}^{n}\left(V_{1 m} h_{1}(n-m)+V_{2 m} h_{2}(n-m)+V_{3 m} h_{3}(n-m)+V_{4 m} h_{4}(n-m)\right. \\
& \left.+V_{5 m} h_{5}(n-m)+V_{6 m} h_{6}(n-m)+V_{7 m} h_{7}(n-m)\right),
\end{aligned}
$$

the stationary zero curvature equation (3.2) can be decomposed into

$$
-V_{+x}^{(n)}+\left[U, V_{+}^{(n)}\right]=V_{-x}^{(n)}-\left[U, V_{-}^{(n)}\right]
$$

Simple computation results in

$$
\begin{aligned}
-V_{+x}^{(n)}+\left[U, V_{+}^{(n)}\right]= & 2 V_{3, n+1} h_{1}(0)+2 V_{1, n+1} h_{3}(0)-V_{5, n+1} h_{4}(0)-V_{4, n+1} h_{5}(0) \\
& -V_{7, n+1} h_{6}(0)-V_{6, n+1} h_{7}(0) .
\end{aligned}
$$

Taking $V^{(n)}=V_{+}^{(n)}-\frac{1}{r} V_{3, n+1} h_{2}(0)$, we have

$$
\begin{aligned}
-V_{x}^{(n)}+\left[U, V^{(n)}\right]= & \left(\frac{1}{r} V_{3, n+1}\right)_{x} h_{2}(0)-\frac{1}{r} V_{2, n+1 x} h_{3}(0) \\
& +\left(-V_{5, n+1}+\frac{u_{2}}{r} V_{3, n+1}\right) h_{4}(0)
\end{aligned}
$$




$$
\begin{aligned}
& +\left(-V_{4, n+1}+\frac{u_{1}}{r} V_{3, n+1}\right) h_{5}(0)+\left(-V_{7, n+1}+\frac{s_{2}}{r} V_{3, n+1}\right) h_{6}(0) \\
& +\left(-V_{6, n+1}+\frac{s_{1}}{r} V_{3, n+1}\right) h_{7}(0) .
\end{aligned}
$$

Hence the zero curvature equation

$$
U_{t}-V_{x}^{(n)}+\left[U, V^{(n)}\right]=0
$$

gives rise to the Lax integrable hierarchy

$$
u_{t}=\left(\begin{array}{c}
q \\
r \\
u_{1} \\
u_{2} \\
s_{1} \\
s_{2}
\end{array}\right)_{t}=\left(\begin{array}{c}
-\partial\left(\frac{1}{r} V_{3, n+1}\right) \\
\frac{1}{r} \partial\left(V_{2, n+1}\right) \\
V_{5, n+1}-\frac{u_{2}}{r} V_{3, n+1} \\
V_{4, n+1}-\frac{u_{1}}{r} V_{3, n+1} \\
V_{7, n+1}-\frac{s_{2}}{r} V_{3, n+1} \\
V_{6, n+1}-\frac{s_{1}}{r} V_{3, n+1}
\end{array}\right) .
$$

When we take $s_{1}=s_{2}=0$, (3.5) can be reduced to an integrable coupling of the generalized coupled Burgers equation hierarchy,

$$
u_{t}=\left(\begin{array}{c}
q \\
r \\
u_{1} \\
u_{2}
\end{array}\right)_{t}=\left(\begin{array}{c}
-\partial\left(\frac{1}{r} V_{3, n+1}\right) \\
\frac{1}{r} \partial\left(V_{2, n+1}\right) \\
V_{5, n+1}-\frac{u_{2}}{r} V_{3, n+1} \\
V_{4, n+1}-\frac{u_{1}}{r} V_{3, n+1}
\end{array}\right) .
$$

And (3.4) can be reduced to another generalized coupled Burgers equation hierarchy by taking $u_{1}=u_{2}=0$,

$$
u_{t}=\left(\begin{array}{c}
q \\
r \\
s_{1} \\
s_{2}
\end{array}\right)_{t}=\left(\begin{array}{c}
-\partial\left(\frac{1}{r} V_{3, n+1}\right) \\
\frac{1}{r} \partial\left(V_{2, n+1}\right) \\
V_{7, n+1}-\frac{s_{2}}{r} V_{3, n+1} \\
V_{6, n+1}-\frac{s_{1}}{r} V_{3, n+1}
\end{array}\right) \text {. }
$$

So we call (3.5) the first kind of coupling integrable couplings of the generalized coupled Burgers equation hierarchy.

When we set $V_{1,0}=0, V_{2,0}=\beta, V_{3,0}=V_{4,0}=V_{5,0}=V_{6,0}=V_{7,0}=0$, we can obtain $V_{1,1}=\beta$, $V_{2,1}=0, V_{3,1}=\beta r, V_{4,1}=\beta u_{1}, V_{5,1}=\beta u_{2}, V_{6,1}=\beta s_{1}, V_{7,1}=\beta s_{2}, \ldots$.

Hence, when we take $n=2$ in Eq. (3.5), we obtain the first kind of the coupling integrable couplings of the generalized coupled Burgers equation, that is,

$$
\left\{\begin{array}{l}
q_{t}=-\beta\left(\frac{r_{x x}}{4 r}+\frac{q_{x}}{r}+q^{2}+\frac{r^{2}}{2}\right)_{x}, \\
r_{t}=\beta\left(\frac{r_{x x}}{2 r}+\frac{q_{x}}{r}-q_{x} r-2 q r_{x}\right), \\
u_{1 t}=\beta u_{2 x x}-2 \beta u_{1 x} q-\beta u_{1} q_{x}-\beta u_{2 x}-\frac{\beta}{2} u_{1} r_{x}-\beta u_{1 x} r-\beta \frac{u_{2} r_{x x}}{4 r}-\beta \frac{u_{2} q_{x}}{2 r}, \\
u_{2 t}=\beta u_{1 x x}-2 \beta u_{2 x} q-\beta u_{2} q_{x}+\beta u_{1 x}+\frac{\beta}{2} u_{2} r_{x}+\beta u_{2 x} r-\beta \frac{u_{1} r_{x x}}{4 r}-\beta \frac{u_{1} q_{x}}{2 r}, \\
s_{1 t}=\beta s_{2 x x}-2 \beta s_{1 x} q-\beta s_{1} q_{x}-\beta s_{2 x}-\frac{\beta}{2} s_{1} r_{x}-\beta s_{1 x} r-\beta \frac{s_{2} r_{x x}}{4 r}-\beta \frac{s_{2} q_{x}}{2 r}, \\
s_{2 t}=\beta s_{1 x x}-2 \beta s_{2 x} q-\beta s_{2} q_{x}+\beta s_{1 x}+\frac{\beta}{2} s_{2} r_{x}+\beta s_{2 x} r-\beta \frac{s_{1} r_{x x}}{4 r}-\beta \frac{s_{1} q_{x}}{2 r} .
\end{array}\right.
$$


When we take $u_{1}=u_{2}=s_{1}=s_{2}=0, \beta=-2$ in (3.9), we can get equations as follows:

$$
\left\{\begin{array}{l}
q_{t}=\left(\frac{r_{x x}}{2 r}+\frac{q_{x}}{r}+2 q^{2}+r^{2}\right)_{x} \\
r_{t}=-\frac{r_{x x}}{r} q_{x x}-\frac{2 q_{x}}{r}+2 q_{x} r+4 q r_{x}
\end{array}\right.
$$

And when we take $r=-1$ in (3.8), we can get the famous generalized Burgers equation

$$
q_{t}=4 q q_{x}-q_{x x}
$$

(II) The second kind of coupling integrable couplings of the generalized coupled Burgers equation hierarchy.

We have the loop algebra of the Lie algebra $F$ :

$$
\begin{aligned}
& \widetilde{F}=\operatorname{span}\left\{f_{i}(n)=f_{i} \lambda^{n}, i=1,2,3,4,5,6,7,8,9\right\}, \\
& {\left[f_{i}(m), f_{j}(n)\right]=\left[f_{i}, f_{j}\right] \lambda^{m+n}, \quad m, n \in Z, i, j=1,2,3,4,5,6,7,8,9,}
\end{aligned}
$$

of which the resulting commutators are defined the same as (2.2).

By using the loop algebra $\widetilde{F}$, we introduce an isospectral Lax pair for zero curvature equation as follows:

$$
\left\{\begin{aligned}
U= & f_{1}(0)+q f_{2}(0)+f_{2}(1)+r f_{3}(0)+u_{1} f_{4}(0)+u_{2} f_{5}(0)+s_{1} f_{7}(0)+s_{2} f_{9}(0), \\
V= & \sum_{m \geq 0}\left(V_{1 m} f_{1}(-m)+V_{2 m} f_{2}(-m)+V_{3 m} f_{3}(-m)+V_{4 m} f_{4}(-m)\right. \\
& \left.+V_{5 m} f_{5}(-m)+V_{6 m} f_{6}(-m)+V_{7 m} f_{7}(-m)+V_{8 m} f_{8}(-m)+V_{9 m} f_{9}(-m)\right) .
\end{aligned}\right.
$$

The stationary zero curvature

$$
V_{x}=[U, V]
$$

is presented by the recurrence relations as follows:

$$
\left\{\begin{array}{l}
V_{1, m x}=-2 V_{3, m+1}-2 q V_{3 m}+2 r V_{2 m} \\
V_{2, m x}=2 V_{3 m}-2 r V_{1 m} \\
V_{3, m x}=-2 V_{1, m+1}-2 q V_{1 m}+2 V_{2 m} \\
V_{4, m x}=-2 V_{7, m+1}-2 q V_{7 m}+2 s_{1} V_{2 m}+2 r V_{6 m} \\
V_{5, m x}=-2 V_{9, m+1}-2 q V_{9 m}+2 s_{2} V_{2 m}+2 r V_{8 m} \\
V_{6, m x}=2 V_{7 m}-2 s_{1} V_{1 m}+2 u_{1} V_{3 m}-2 r V_{4 m} \\
V_{7, m x}=-2 V_{4, m+1}-2 q V_{4 m}++2 u_{1} V_{2 m}+2 V_{6 m} \\
V_{8, m x}=2 V_{9 m}-2 s_{2} V_{1 m}+2 u_{2} V_{3 m}-2 r V_{5 m} \\
V_{9, m x}=-2 V_{5, m+1}-2 q V_{5 m}+2 u_{2} V_{2 m}+2 V_{8 m} .
\end{array}\right.
$$

Noting that

$$
V_{+}^{(n)}=\sum_{m=0}^{n}\left(V_{1 m} f_{1}(n-m)+V_{2 m} f_{2}(n-m)+V_{3 m} f_{3}(n-m)+V_{4 m} f_{4}(n-m)\right.
$$




$$
\left.+V_{5 m} f_{5}(n-m)+V_{6 m} f_{6}(n-m)+V_{7 m} f_{7}(n-m)+V_{8 m} f_{8}(n-m)+V_{9 m} f_{9}(n-m)\right),
$$

we obtain by a direct calculation

$$
\begin{aligned}
-V_{+x}^{(n)}+\left[U, V_{+}^{(n)}\right]= & 2 V_{3, n+1} f_{1}(0)+2 V_{1, n+1} f_{3}(0)+2 V_{7, n+1} f_{4}(0)+2 V_{9, n+1} f_{5}(0) \\
& +2 V_{4, n+1} f_{7}(0)+2 V_{5, n+1} f_{9}(0) .
\end{aligned}
$$

Taking $V^{(n)}=V_{+}^{(n)}-\frac{1}{r} V_{3, n+1} f_{2}(0)$, after a calculation, we get

$$
\begin{aligned}
-V_{x}^{(n)}+\left[U, V^{(n)}\right]= & \left(\frac{1}{r} V_{3, n+1}\right)_{x} f_{2}(0)-\frac{1}{r} V_{2, n+1 x} f_{3}(0)+\left(2 V_{7, n+1}-\frac{2 s_{1}}{r} V_{3, n+1}\right) f_{4}(0) \\
& +\left(2 V_{9, n+1}-\frac{2 s_{2}}{r} V_{3, n+1}\right) f_{5}(0)+\left(2 V_{4, n+1}-\frac{2 u_{1}}{r} V_{3, n+1}\right) f_{7}(0) \\
& +\left(2 V_{5, n+1}-\frac{2 u_{2}}{r} V_{3, n+1}\right) f_{9}(0) .
\end{aligned}
$$

The zero curvature equation

$$
U_{t}-V_{x}^{(n)}+\left[U, V^{(n)}\right]=0
$$

admits

$$
u_{t}=\left(\begin{array}{c}
q \\
r \\
u_{1} \\
u_{2} \\
s_{1} \\
s_{2}
\end{array}\right)_{t}=\left(\begin{array}{c}
-\left(\frac{1}{r} V_{3, n+1}\right)_{x} \\
\frac{1}{r} V_{2, n+1 x} \\
-2 V_{7, n+1}+\frac{2 s_{1}}{r} V_{3, n+1} \\
-2 V_{9, n+1}+\frac{2 s_{2}}{r} V_{3, n+1} \\
-2 V_{4, n+1}+\frac{2 u_{1}}{r} V_{3, n+1} \\
-2 V_{5, n+1}+\frac{2 u_{2}}{r} V_{3, n+1}
\end{array}\right),
$$

which can be regarded as a composition of two integrable coupling of the generalized coupled Burgers equation hierarchy as follows:

$$
\begin{aligned}
& u_{t}=\left(\begin{array}{l}
q \\
r \\
u_{1} \\
u_{2}
\end{array}\right)_{t}=\left(\begin{array}{c}
-\left(\frac{1}{r} V_{3, n+1}\right)_{x} \\
\frac{1}{r} V_{2, n+1 x} \\
-2 V_{7, n+1}+\frac{2 s_{1}}{r} V_{3, n+1} \\
-2 V_{9, n+1}+\frac{2 s_{2}}{r} V_{3, n+1}
\end{array}\right), \\
& u_{t}=\left(\begin{array}{c}
q \\
r \\
s_{1} \\
s_{2}
\end{array}\right)_{t}=\left(\begin{array}{c}
-\left(\frac{1}{r} V_{3, n+1}\right)_{x} \\
\frac{1}{r} V_{2, n+1 x} \\
-2 V_{4, n+1}+\frac{2 u_{1}}{r} V_{3, n+1} \\
-2 V_{5, n+1}+\frac{2 u_{2}}{r} V_{3, n+1}
\end{array}\right) .
\end{aligned}
$$

When we set $V_{1,0}=0, V_{2,0}=\beta, V_{3,0}=V_{4,0}=V_{5,0}=V_{6,0}=V_{7,0}=V_{8,0}=V_{9,0}=0$, we can obtain $V_{1,1}=\beta, V_{2,1}=0, V_{3,1}=\beta r, V_{4,1}=\beta u_{1}, V_{5,1}=\beta u_{2}, V_{6,1}=0, V_{7,1}=\beta s_{1}, V_{8,1}=0$, $V_{9,1}=\beta s_{2}, \ldots$. 
Hence, when we take $n=2$ in (3.14), we get the second kind of the coupling integrable couplings of the generalized coupled Burgers equation as follows:

$$
\left\{\begin{array}{l}
q_{t}=-\beta\left(\frac{r_{x x}}{4 r}+\frac{q_{x}}{r}+q^{2}+\frac{r^{2}}{2}\right)_{x}, \\
r_{t}=\beta\left(\frac{r_{x x}}{2 r}+\frac{q_{x}}{r}-q_{x} r-2 q r_{x}\right), \\
u_{1 t}=-\frac{\beta}{2} s_{1 x x}-2 \beta u_{1 x} q-\beta u_{1} q_{x}+2 \beta u_{1} r-2 \beta s_{1} r^{2}+\frac{\beta}{2} \frac{s_{1} r_{x x}}{r}+\beta \frac{s_{1} q_{x}}{r} \\
u_{2 t}=-\frac{\beta}{2} s_{2 x x}-2 \beta u_{2 x} q-\beta u_{2} q_{x}+2 \beta u_{2} r-2 \beta s_{2} r^{2}+\frac{\beta}{2} \frac{s_{2} r_{x x}}{r}+\beta \frac{s_{2} q_{x}}{r} \\
s_{1 t}=-\frac{\beta}{2} u_{1 x x}-2 \beta s_{1 x} q-\beta s_{1} q_{x}+2 \beta u_{1}-2 \beta s_{1} r+\frac{\beta}{2} \frac{u_{1} r_{x x}}{r}+\beta \frac{u_{1} q_{x}}{r} \\
s_{2 t}=-\frac{\beta}{2} u_{2 x x}-2 \beta s_{2 x} q-\beta s_{2} q_{x}+2 \beta u_{2}-2 \beta s_{2} r+\frac{\beta}{2} \frac{u_{2} r_{x x}}{r}+\beta \frac{u_{2} q_{x}}{r} .
\end{array}\right.
$$

When take $u_{1}=u_{2}=s_{1}=s_{2}=0, r=-1, \beta=-2$ in (3.17), we can also get the same as (3.10).

(III) The third kind of coupling integrable couplings of the generalized coupled Burgers equation hierarchy.

By using the loop algebra of Lie algebra $H$ as follows:

$$
\widetilde{H}=\operatorname{span}\left\{f_{i}(n)=f_{i} \lambda^{n}, i=1,2,3,4,5,6,7,8,9\right\},
$$

along with the commutator

$$
\left[f_{i}(m), f_{j}(n)\right]=\left[f_{i}, f_{j}\right] \lambda^{m+n}, \quad m, n \in Z, i, j=1,2,3,4,5,6,7,8,9,
$$

we get the third type of coupling integrable couplings of the generalized coupled Burgers equation hierarchy.

A Lax pair for zero curvature equation is given as follows:

$$
\left\{\begin{aligned}
U= & f_{1}(0)+q f_{2}(0)+f_{2}(1)+r f_{3}(0)+u_{1} f_{4}(0)+u_{2} f_{6}(0)+s_{1} f_{7}(0)+s_{2} f_{9}(0) \\
V= & \sum_{m \geq 0}\left(V_{1 m} f_{1}(-m)+V_{2 m} f_{2}(-m)+V_{3 m} f_{3}(-m)+V_{4 m} f_{4}(-m)\right. \\
& \left.+V_{5 m} f_{5}(-m)+V_{6 m} f_{6}(-m)+V_{7 m} f_{7}(-m)+V_{8 m} f_{8}(-m)+V_{9 m} f_{9}(-m)\right)
\end{aligned}\right.
$$

A solution to the stationary zero curvature equation

$$
V_{x}=[U, V]
$$

is presented now:

$$
\left\{\begin{array}{l}
V_{1, m x}=-2 V_{3, m+1}-2 q V_{3 m}+2 r V_{2 m} \\
V_{2, m x}=2 V_{3 m}-2 r V_{1 m} \\
V_{3, m x}=-2 V_{1, m+1}-2 q V_{1 m}+2 V_{2 m} \\
V_{4, m x}=-2 V_{6, m+1}-2 q V_{6 m}+2 r V_{5 m}+2 u_{2} V_{2 m} \\
V_{5, m x}=2 V_{6 m}-2 u_{2} V_{1 m}+2 u_{1} V_{3 m}-2 r V_{4 m} \\
V_{6, m x}=-2 V_{4, m+1}-2 q V_{4 m}+2 V_{5 m}+2 u_{1} V_{2 m} \\
V_{7, m x}=-2 V_{9, m+1}-2 q V_{9 m}+2 r V_{8 m}+2 s_{2} V_{2 m}+2 s_{2} V_{8 m} \\
V_{8, m x}=2 V_{9 m}-2 s_{2} V_{1 m}+2 s_{1} V_{3 m}-2 r V_{7 m}+2 s_{1} V_{9 m}-2 s_{2} V_{7 m} \\
V_{9, m x}=-2 V_{7, m+1}-2 q V_{7 m}+2 V_{8 m}+2 s_{1} V_{2 m}+2 s_{1} V_{8 m}
\end{array}\right.
$$


Setting

$$
\begin{aligned}
V_{+}^{(n)}= & \sum_{m=0}^{n}\left(V_{1 m} f_{1}(n-m)+V_{2 m} f_{2}(n-m)+V_{3 m} f_{3}(n-m)+V_{4 m} f_{4}(n-m)\right. \\
& \left.+V_{5 m} f_{5}(n-m)+V_{6 m} f_{6}(n-m)+V_{7 m} f_{7}(n-m)+V_{8 m} f_{8}(n-m)+V_{9 m} f_{9}(n-m)\right),
\end{aligned}
$$

we have

$$
\begin{aligned}
-V_{+x}^{(n)} & +\left[U, V_{+}^{(n)}\right] \\
= & 2 V_{3, n+1} f_{1}(0)+2 V_{1, n+1} f_{3}(0)+2 V_{6, n+1} f_{4}(0) \\
& +2 V_{4, n+1} f_{6}(0)+2 V_{9, n+1} f_{7}(0)+2 V_{7, n+1} f_{9}(0) .
\end{aligned}
$$

Taking $V^{(n)}=V_{+}^{(n)}-\frac{1}{r} V_{3, n+1} f_{2}(0)$, we obtain

$$
\begin{aligned}
-V_{x}^{(n)}+\left[U, V^{(n)}\right]= & \left(\frac{1}{r} V_{3, n+1}\right)_{x} f_{2}(0)-\frac{1}{r} V_{2, n+1} f_{3}(0)+\left(2 V_{6, n+1}-2 \frac{u_{2}}{r} V_{3, n+1}\right) f_{4}(0) \\
& +\left(2 V_{4, n+1}-2 \frac{u_{1}}{r} V_{3, n+1}\right) f_{6}(0)+\left(2 V_{9, n+1}-2 \frac{s_{2}}{r} V_{3, n+1}\right) f_{7}(0) \\
& +\left(2 V_{7, n+1}-2 \frac{s_{1}}{r} V_{3, n+1}\right) f_{9}(0) .
\end{aligned}
$$

Thus, the zero curvature equation

$$
U_{t}-V_{x}^{(n)}+\left[U, V^{(n)}\right]=0
$$

admits the Lax integrable hierarchy

$$
u_{t}=\left(\begin{array}{c}
q \\
r \\
u_{1} \\
u_{2} \\
s_{1} \\
s_{2}
\end{array}\right)_{t}=\left(\begin{array}{c}
-\left(\frac{1}{r} V_{3, n+1}\right)_{x} \\
\frac{1}{r} V_{2, n+1 x} \\
-2 V_{6, n+1}+2 \frac{u_{2}}{r} V_{3, n+1} \\
-2 V_{4, n+1}+2 \frac{u_{1}}{r} V_{3, n+1} \\
-2 V_{9, n+1}+2 \frac{s_{2}}{r} V_{3, n+1} \\
-2 V_{7, n+1}+2 \frac{s_{1}}{r} V_{3, n+1}
\end{array}\right) \text {. }
$$

When we take $s_{1}=s_{2}=0$ and $u_{1}=u_{2}=0$ in (3.21), respectively, we get two integrable coupling of the generalized coupled Burgers equation hierarchy

$$
\begin{aligned}
& u_{t}=\left(\begin{array}{l}
q \\
r \\
u_{1} \\
u_{2}
\end{array}\right)_{t}=\left(\begin{array}{c}
-\left(\frac{1}{r} V_{3, n+1}\right)_{x} \\
\frac{1}{r} V_{2, n+1 x} \\
-2 V_{6, n+1}+2 \frac{u_{2}}{r} V_{3, n+1} \\
-2 V_{4, n+1}+2 \frac{u_{1}}{r} V_{3, n+1}
\end{array}\right), \\
& u_{t}=\left(\begin{array}{c}
q \\
r \\
s_{1} \\
s_{2}
\end{array}\right)_{t}=\left(\begin{array}{c}
-\left(\frac{1}{r} V_{3, n+1}\right)_{x} \\
\frac{1}{r} V_{2, n+1 x} \\
-2 V_{9, n+1}+2 \frac{s_{2}}{r} V_{3, n+1} \\
-2 V_{7, n+1}+2 \frac{s_{1}}{r} V_{3, n+1}
\end{array}\right) .
\end{aligned}
$$


So we call (3.21) the third type of coupling integrable couplings of the generalized coupled Burgers equation hierarchy.

Set $V_{1,0}=0, V_{2,0}=\beta, V_{3,0}=V_{4,0}=V_{5,0}=V_{6,0}=V_{7,0}=V_{8,0}=V_{9,0}=0$, we can obtain $V_{1,1}=$ $\beta, V_{2,1}=0, V_{3,1}=\beta r, V_{4,1}=\beta u_{1}, V_{5,1}=0, V_{6,1}=\beta u_{2}, V_{7,1}=\beta s_{1}, V_{8,1}=0, V_{9,1}=\beta s_{2}, \ldots$

And when we take $n=2$ in (3.21), we get the third hind of the coupling integrable couplings of the generalized coupled Burgers equation, that is,

$$
\left\{\begin{aligned}
q_{t}= & -\beta\left(\frac{r_{x x}}{4 r}+\frac{q_{x}}{r}+q^{2}+\frac{r^{2}}{2}\right)_{x} \\
r_{t}= & \beta\left(\frac{r_{x x}}{2 r}+\frac{q_{x}}{r}-q_{x} r-2 q r_{x}\right) \\
u_{1 t}= & -\frac{\beta}{2} u_{2 x x}-2 \beta u_{1 x} q-\beta u_{1} q_{x}+2 \beta u_{1} r-2 \beta u_{2} r^{2}+\frac{\beta}{2} \frac{u_{2} r_{x x}}{r}+\beta \frac{u_{2} q_{x}}{r} \\
u_{2 t}= & -\frac{\beta}{2} u_{1 x x}-2 \beta u_{2 x} q-\beta u_{2} q_{x}+2 \beta u_{1}-2 \beta u_{2} r+\frac{\beta}{2} \frac{u_{1} r_{x x}}{r}+\beta \frac{u_{1} q_{x}}{r} \\
s_{1 t}= & -\frac{\beta}{2} s_{2 x x}-2 \beta s_{1 x} q-\beta s_{1} q_{x}+2 \beta s_{1} r-2 \beta s_{2} r^{2}-3 \beta s_{2}^{2} r+\beta s_{1}^{2} r+2 \beta s_{1} s_{2} \\
& \quad-\beta s_{2}^{3}+\beta s_{1}^{2} s_{2}+\frac{\beta}{2} \frac{s_{2} r_{x x}}{r}+\beta \frac{s_{2} q_{x}}{r} \\
s_{2 t}= & -\frac{\beta}{2} s_{1 x x}-2 \beta s_{2 x} q-\beta s_{2} q_{x}+2 \beta s_{1}-2 \beta s_{2} r+3 \beta s_{1}^{2} r-\beta s_{2}^{2}-2 \beta s_{1} s_{2} r \\
& +\beta s_{1}^{3}-\beta s_{1} s_{2}^{2}+\frac{\beta}{2} \frac{s_{1} r_{x x}}{r}+\beta \frac{s_{1} q_{x}}{r} .
\end{aligned}\right.
$$

Similarly when take $u_{1}=u_{2}=s_{1}=s_{2}=0, n=2, \alpha=-2$ in (3.24), we can obtain (3.10).

\section{Hamiltonian structure of coupling integrable couplings of the generalized coupled Burgers equation hierarchy}

In this section we shall deduce the Hamiltonian forms of the coupling integrable couplings (3.21) by using the quadratic-form [39]. The commutator (2.4) can be written as

$$
[a, b]^{T}=a^{T} R(b)
$$

where

$R(b)$

$$
=\left(\begin{array}{ccccccccc}
0 & 2 b_{3} & 2 b_{2} & 0 & 2 b_{6} & 2 b_{5} & 0 & 2 b_{9} & 2 b_{8} \\
-2 b_{3} & 0 & -2 b_{1} & -2 b_{6} & 0 & -2 b_{4} & -2 b_{9} & 0 & -2 b_{7} \\
2 b_{2} & -2 b_{1} & 0 & 2 b_{5} & -2 b_{4} & 0 & 2 b_{8} & -2 b_{7} & 0 \\
0 & 0 & 0 & 0 & 2 b_{3} & 2 b_{2} & 0 & 0 & 0 \\
0 & 0 & 0 & -2 b_{3} & 0 & -2 b_{1} & 0 & 0 & 0 \\
0 & 0 & 0 & 2 b_{2} & -2 b_{1} & 0 & 0 & 0 & 0 \\
0 & 0 & 0 & 0 & 0 & 0 & 0 & 2 b_{3}+2 b_{9} & 2 b_{2}+2 b_{8} \\
0 & 0 & 0 & 0 & 0 & 0 & -2 b_{3}-2 b_{9} & 0 & -2 b_{1}-2 b_{7} \\
0 & 0 & 0 & 0 & 0 & 0 & 2 b_{2}+2 b_{8} & -2 b_{1}-2 b_{7} & 0
\end{array}\right) .
$$

The constant symmetric matrix $F$ satisfying the matrix equation

$$
R(b) F=-(R(b) F)^{T}, \quad F=F^{T},
$$


shows that

$$
F=\left(\begin{array}{ccccccccc}
1 & 0 & 0 & 1 & 0 & 0 & 1 & 0 & 0 \\
0 & 1 & 0 & 0 & 1 & 0 & 0 & 1 & 0 \\
0 & 0 & -1 & 0 & 0 & -1 & 0 & 0 & -1 \\
1 & 0 & 0 & 0 & 0 & 0 & 0 & 0 & 0 \\
0 & 1 & 0 & 0 & 0 & 0 & 0 & 0 & 0 \\
0 & 0 & -1 & 0 & 0 & 0 & 0 & 0 & 0 \\
1 & 0 & 0 & 0 & 0 & 0 & 1 & 0 & 0 \\
0 & 1 & 0 & 0 & 0 & 0 & 0 & 1 & 0 \\
0 & 0 & -1 & 0 & 0 & 0 & 0 & 0 & -1
\end{array}\right) .
$$

In the linear space $R^{9}$, define a functional,

$$
\begin{aligned}
\{a, b\}= & a^{T} F b \\
= & a_{1} b_{1}+a_{2} b_{2}-a_{3} b_{3}+a_{7} b_{7} \\
& +a_{8} b_{8}-a_{9} b_{9}+\left(a_{1} b_{4}+a_{4} b_{1}\right)+\left(a_{1} b_{7}+a_{7} b_{1}\right)+\left(a_{2} b_{5}+a_{5} b_{2}\right) \\
& +\left(a_{2} b_{8}+a_{8} b_{2}\right)-\left(a_{3} b_{6}+a_{6} b_{3}\right)-\left(a_{3} b_{9}+a_{9} b_{3}\right) .
\end{aligned}
$$

The Lax pair $U$ and $V$ in (3.18) can be written as

$$
\left\{\begin{array}{l}
U=\left(1, q+\lambda, r, u_{1}, 0, u_{2}, s_{1}, 0, s_{2}\right)^{T}, \\
V=\left(V_{1}, V_{2}, V_{3}, V_{4}, V_{5}, V_{6}, V_{7}, V_{8}, V_{9}\right),
\end{array}\right.
$$

where $V_{i}=\sum_{m \geq 0} V_{i m} \lambda^{-m}, i=1,2, \ldots, 9$. With the help of (4.2), we have

$$
\begin{aligned}
& \left\{V, \frac{\partial U}{\partial \lambda}\right\}=V_{2}+V_{5}+V_{8}, \\
& \left\{V, \frac{\partial U}{\partial q}\right\}=V_{2}+V_{5}+V_{8}, \\
& \left\{V, \frac{\partial U}{\partial r}\right\}=-V_{3}-V_{6}-V_{9}, \\
& \left\{V, \frac{\partial U}{\partial u_{1}}\right\}=V_{1}, \quad\left\{V, \frac{\partial U}{\partial u_{2}}\right\}=-V_{3}, \\
& \left\{V, \frac{\partial U}{\partial s_{1}}\right\}=V_{1}+V_{7}, \quad\left\{V, \frac{\partial U}{\partial s_{2}}\right\}=-V_{3}-V_{9},
\end{aligned}
$$

substituting the above into the quadratic-form identity yields

$$
\frac{\delta}{\delta \bar{u}}\left(V_{2}+V_{5}+V_{8}\right)=\lambda^{-\gamma} \frac{\partial}{\partial \lambda} \lambda^{\gamma}\left(\begin{array}{c}
V_{2}+V_{5}+V_{8} \\
-V_{3}-V_{6}-V_{9} \\
V_{1} \\
-V_{3} \\
V_{1}+V_{7} \\
-V_{3}-V_{9}
\end{array}\right)
$$


where

$$
\frac{\delta}{\delta u}=\left(\begin{array}{c}
\frac{\delta}{\delta q} \\
\frac{\delta}{\delta r} \\
\frac{\delta}{\delta u_{1}} \\
\frac{\delta}{\delta u_{2}} \\
\frac{\delta}{\delta s_{1}} \\
\frac{\delta}{\delta s_{2}}
\end{array}\right)
$$

Comparing the coefficients of $\lambda^{-n-2}$ on both sides of (4.7), we get

$$
\frac{\delta}{\delta \bar{u}}\left(V_{2, n+2}+V_{5, n+2}+V_{8, n+2}\right)=(\gamma-n-1)\left(\begin{array}{c}
V_{2, n+1}+V_{5, n+1}+V_{8, n+1} \\
-V_{3, n+1}-V_{6, n+1}-V_{9, n+1} \\
V_{1, n+1} \\
-V_{3, n+1} \\
V_{1, n+1}+V_{7, n+1} \\
-V_{3, n+1}-V_{9, n+1}
\end{array}\right) .
$$

Taking $n=0$ in (4.8), we obtain $\gamma=0$.

So we get

$$
\frac{\delta H_{n+2}}{\delta u}=\left(\begin{array}{c}
V_{2, n+1}+V_{5, n+1}+V_{8, n+1} \\
-V_{3, n+1}-V_{6, n+1}-V_{9, n+1} \\
V_{1, n+1} \\
-V_{3, n+1} \\
V_{1, n+1}+V_{7, n+1} \\
-V_{3, n+1}-V_{9, n+1}
\end{array}\right)
$$

where

$$
H_{n+2}=-\frac{V_{2, n+2}+V_{5, n+2}+V_{8, n+2}}{n+1} .
$$

So (3.21) can be written as a Hamiltonian form

$$
\begin{aligned}
u_{t} & =\left(\begin{array}{c}
q \\
r \\
u_{1} \\
u_{2} \\
s_{1} \\
s_{2}
\end{array}\right)_{t}=\left(\begin{array}{c}
-\left(\frac{1}{r} V_{3, n+1}\right)_{x} \\
\frac{1}{r} V_{2, n+1 x} \\
-2 V_{6, n+1}+2 \frac{u_{2}}{r} V_{3, n+1} \\
-2 V_{4, n+1}+2 \frac{u_{1}}{r} V_{3, n+1} \\
-2 V_{9, n+1}+2 \frac{s_{2}}{r} V_{3, n+1} \\
-2 V_{7, n+1}+2 \frac{s_{1}}{r} V_{3, n+1}
\end{array}\right) \\
& =\left(\begin{array}{cccccc}
0 & 0 & 0 & \partial \frac{1}{r} & 0 & 0 \\
0 & 0 & -2 & -\frac{2}{r} & 0 & 0 \\
0 & 2 & 0 & -2 \frac{u_{2}}{r} & 0 & -2 \\
\frac{1}{r} \partial & \frac{2}{r} & 2 \frac{u_{2}}{r} & 0 & 2+2 \frac{s_{2}}{r} & 2 \frac{s_{1}}{r} \\
0 & 0 & 0 & -2-2 \frac{s_{2}}{r} & 0 & 2 \\
0 & 0 & 2 & -2 \frac{s_{1}}{r} & -2 & 0
\end{array}\right)
\end{aligned}
$$




$$
\begin{aligned}
& \times\left(\begin{array}{c}
V_{2, n+1}+V_{5, n+1}+V_{8, n+1} \\
-V_{3, n+1}-V_{6, n+1}-V_{9, n+1} \\
V_{1, n+1} \\
-V_{3, n+1} \\
V_{1, n+1}+V_{7, n+1} \\
-V_{3, n+1}-V_{9, n+1}
\end{array}\right) \\
& =J \frac{\delta H_{n+2}}{\delta u},
\end{aligned}
$$

where $\partial=\frac{\partial}{\partial x}$ and $J$ is a Hamiltonian operator. We can obtain a recursive operator from (3.19),

$$
L=\left(\begin{array}{llllll}
l_{11} & l_{12} & l_{13} & l_{14} & l_{15} & l_{16} \\
l_{21} & l_{22} & l_{23} & l_{24} & l_{25} & l_{26} \\
l_{31} & l_{32} & l_{33} & l_{34} & l_{35} & l_{36} \\
l_{41} & l_{42} & l_{43} & l_{44} & l_{45} & l_{46} \\
l_{51} & l_{52} & l_{53} & l_{54} & l_{55} & l_{56} \\
l_{61} & l_{62} & l_{63} & l_{64} & l_{65} & l_{66}
\end{array}\right)
$$

where

$$
\begin{aligned}
& l_{11}=\frac{1}{2 r} \partial-\partial^{-1} q \partial, \quad l_{12}=\frac{1}{r} \partial-\partial^{-1} r \partial, \quad l_{13}=\frac{u_{2}}{r} \partial-\partial^{-1} u_{1} \partial, \\
& l_{14}=\frac{u_{1}}{r} \partial-\partial^{-1} u_{2} \partial, \quad l_{15}=\frac{s_{2}}{r} \partial-\partial^{-1} s_{1} \partial, \quad l_{16}=\frac{s_{1}}{r} \partial-\partial^{-1} s_{2} \partial, \\
& l_{21}=-r-\frac{1}{4} \partial \frac{1}{r} \partial, \quad l_{22}=-q-\frac{1}{2} \partial \frac{1}{r}, \quad l_{23}=2 u_{2} \partial^{-1} r-\frac{1}{2} \partial \frac{u_{2}}{r}, \quad l_{24}=-\frac{1}{2} \partial \frac{u_{1}}{r}, \\
& l_{25}=2 s_{2} \partial^{-1} r+2 s_{2} \partial^{-1} s_{2}-\frac{1}{2} \partial \frac{s_{2}}{r}, \quad l_{26}=-\frac{1}{2} \partial \frac{s_{1}}{r}+2 s_{2} \partial^{-1}+2 s_{2} \partial^{-1} s_{1}, \\
& l_{31}=l_{32}=l_{35}=l_{36}=0, \quad l_{33}=-q-2 \partial^{-1} r, \quad l_{34}=\frac{\partial}{2}-2 \partial^{-1}, \\
& l_{41}=l_{42}=l_{45}=l_{46}=0, \quad l_{43}=\frac{\partial}{2}+2 r \partial^{-1} r, \quad l_{34}=2 r \partial^{-1}-q, \\
& l_{51}=l_{52}=l_{53}=l_{54}=0, \quad l_{55}=-q-2 \partial^{-1} r-2 \partial^{-1} s_{2}-2 s_{1} \partial^{-1} r-2 s_{1} \partial^{-1} s_{2}, \\
& l_{56}=\frac{1}{2} \partial-2 \partial^{-1}-2 \partial^{-1} s_{1}-2 s_{1} \partial^{-1}-2 s_{1} \partial^{-1} s_{1}, \\
& l_{61}=l_{62}=l_{63}=l_{64}=0, \quad l_{65}=\frac{1}{2} \partial+2 r \partial^{-1} r+2 r \partial^{-1} s_{2}+2 s_{2} \partial^{-1} r+2 s_{2} \partial^{-1} s_{2}, \\
& l_{66}=-q+2 r \partial^{-1}+2 r \partial^{-1} s_{1}+2 s_{2} \partial^{-1}+2 s_{2} \partial^{-1} s_{1} .
\end{aligned}
$$

$L$ satisfies

$$
\left(\begin{array}{c}
V_{2, n+1}+V_{5, n+1}+V_{8, n+1} \\
-V_{3, n+1}-V_{6, n+1}-V_{9, n+1} \\
V_{1, n+1} \\
-V_{3, n+1} \\
V_{1, n+1}+V_{7, n+1} \\
-V_{3, n+1}-V_{9, n+1}
\end{array}\right)=L\left(\begin{array}{c}
V_{2 n}+V_{5 n}+V_{8 n} \\
-V_{3 n}-V_{6 n}-V_{9 n} \\
V_{1 n} \\
-V_{3 n} \\
V_{1 n}+V_{7 n} \\
-V_{3 n}-V_{9 n}
\end{array}\right) .
$$


The Hamiltonian form of the coupling integrable couplings (3.21) can be written as

$$
u_{t}=J \frac{\delta H_{n+2}}{\delta u}=J L^{n}\left(\begin{array}{c}
0 \\
-\beta r-\beta u_{2}-\beta s_{2} \\
\beta \\
-\beta r \\
\beta+\beta s_{1} \\
-\beta r-\beta s_{2}
\end{array}\right) \text {. }
$$

\section{Conclusion}

In this paper we get three kinds of coupling integrable couplings of the generalized coupled Burgers equation hierarchy, which are new results. And we obtain the Hamiltonian structure of one coupling integrable couplings of the generalized coupled Burgers equation hierarchy by using the quadratic-form identity.

\section{Results and discussion}

Firstly, we introduce three Lie algebras $E, F$ and $H$. With the help of their corresponding loop algebras $\widetilde{E}, \widetilde{F}$, and $\widetilde{H}$, we establish three isospectral problems, respectively. Then, by taking advantage of the Tu scheme, we get three kinds of coupling integrable couplings of the generalized coupled Burgers equation hierarchy as follows:

$$
\begin{aligned}
& \left\{\begin{aligned}
q_{t}= & -\beta\left(\frac{r_{x x}}{4 r}+\frac{q_{x}}{r}+q^{2}+\frac{r^{2}}{2}\right)_{x}, \\
r_{t}= & \beta\left(\frac{r_{x x}}{2 r}+\frac{q_{x}}{r}-q_{x} r-2 q r_{x}\right), \\
u_{1 t}= & -\frac{\beta}{2} u_{2 x x}-2 \beta u_{1 x} q-\beta u_{1} q_{x}+2 \beta u_{1} r-2 \beta u_{2} r^{2}+\frac{\beta}{2} \frac{u_{2} r_{x x}}{r}+\beta \frac{u_{2} q_{x}}{r}, \\
u_{2 t}= & -\frac{\beta}{2} u_{1 x x}-2 \beta u_{2 x} q-\beta u_{2} q_{x}+2 \beta u_{1}-2 \beta u_{2} r+\frac{\beta}{2} \frac{u_{1} r_{x x}}{r}+\beta \frac{u_{1} q_{x}}{r}, \\
s_{1 t}= & -\frac{\beta}{2} s_{2 x x}-2 \beta s_{1 x} q-\beta s_{1} q_{x}+2 \beta s_{1} r-2 \beta s_{2} r^{2}-3 \beta s_{2}^{2} r+\beta s_{1}^{2} r+2 \beta s_{1} s_{2} \\
& -\beta s_{2}^{3}+\beta s_{1}^{2} s_{2}+\frac{\beta}{2} \frac{s_{2} r_{x x}}{r}+\beta \frac{s_{2} q_{x}}{r}, \\
s_{2 t}= & -\frac{\beta}{2} s_{1 x x}-2 \beta s_{2 x} q-\beta s_{2} q_{x}+2 \beta s_{1}-2 \beta s_{2} r+3 \beta s_{1}^{2} r-\beta s_{2}^{2}-2 \beta s_{1} s_{2} r+\beta s_{1}^{3} \\
& -\beta s_{1} s_{2}^{2}+\frac{\beta}{2} \frac{s_{1} r_{x x}}{r}+\beta \frac{s_{1} q_{x}}{r},
\end{aligned}\right. \\
& \left\{\begin{array}{l}
q_{t}=-\beta\left(\frac{r_{x x}}{4 r}+\frac{q_{x}}{r}+q^{2}+\frac{r^{2}}{2}\right)_{x}, \\
r_{t}=\beta\left(\frac{r_{x x}}{2 r}+\frac{q_{x}}{r}-q_{x} r-2 q r_{x}\right), \\
u_{1 t}=\beta u_{2 x x}-2 \beta u_{1 x} q-\beta u_{1} q_{x}-\beta u_{2 x}-\frac{\beta}{2} u_{1} r_{x}-\beta u_{1 x} r-\beta \frac{u_{2} r_{x x}}{4 r}-\beta \frac{u_{2} q_{x}}{2 r}, \\
u_{2 t}=\beta u_{1 x x}-2 \beta u_{2 x} q-\beta u_{2} q_{x}+\beta u_{1 x}+\frac{\beta}{2} u_{2} r_{x}+\beta u_{2 x} r-\beta \frac{u_{1} r_{x x}}{4 r}-\beta \frac{u_{1} q_{x}}{2 r} \\
s_{1 t}=\beta s_{2 x x}-2 \beta s_{1 x} q-\beta s_{1} q_{x}-\beta s_{2 x}-\frac{\beta}{2} s_{1} r_{x}-\beta s_{1 x} r-\beta \frac{s_{2} r_{x x}}{4 r}-\beta \frac{s_{2} q_{x}}{2 r} \\
s_{2 t}=\beta s_{1 x x}-2 \beta s_{2 x} q-\beta s_{2} q_{x}+\beta s_{1 x}+\frac{\beta}{2} s_{2} r_{x}+\beta s_{2 x} r-\beta \frac{s_{1} r_{x x}}{4 r}-\beta \frac{s_{1} q_{x}}{2 r},
\end{array}\right. \\
& \left\{\begin{array}{l}
q_{t}=-\beta\left(\frac{r_{x x}}{4 r}+\frac{q_{x}}{r}+q^{2}+\frac{r^{2}}{2}\right)_{x}, \\
r_{t}=\beta\left(\frac{r_{x x}}{2 r}+\frac{q_{x}}{r}-q_{x} r-2 q r_{x}\right), \\
u_{1 t}=-\frac{\beta}{2} s_{1 x x}-2 \beta u_{1 x} q-\beta u_{1} q_{x}+2 \beta u_{1} r-2 \beta s_{1} r^{2}+\frac{\beta}{2} \frac{s_{1} r_{x x}}{r}+\beta \frac{s_{1} q_{x}}{r}, \\
u_{2 t}=-\frac{\beta}{2} s_{2 x x}-2 \beta u_{2 x} q-\beta u_{2} q_{x}+2 \beta u_{2} r-2 \beta s_{2} r^{2}+\frac{\beta}{2} \frac{s_{2} r_{x x}}{r}+\beta \frac{s_{2} q_{x}}{r}, \\
s_{1 t}=-\frac{\beta}{2} u_{1 x x}-2 \beta s_{1 x} q-\beta s_{1} q_{x}+2 \beta u_{1}-2 \beta s_{1} r+\frac{\beta}{2} \frac{u_{1} r_{x x}}{r}+\beta \frac{u_{1} q_{x}}{r}, \\
s_{2 t}=-\frac{\beta}{2} u_{2 x x}-2 \beta s_{2 x} q-\beta s_{2} q_{x}+2 \beta u_{2}-2 \beta s_{2} r+\frac{\beta}{2} \frac{u_{2} r_{x x}}{r}+\beta \frac{u_{2} q_{x}}{r},
\end{array}\right.
\end{aligned}
$$




$$
\left\{\begin{aligned}
q_{t}= & -\beta\left(\frac{r_{x x}}{4 r}+\frac{q_{x}}{r}+q^{2}+\frac{r^{2}}{2}\right)_{x} \\
r_{t}= & \beta\left(\frac{r_{x x}}{2 r}+\frac{q_{x}}{r}-q_{x} r-2 q r_{x}\right), \\
u_{1 t}= & -\frac{\beta}{2} u_{2 x x}-2 \beta u_{1 x} q-\beta u_{1} q_{x}+2 \beta u_{1} r-2 \beta u_{2} r^{2}+\frac{\beta}{2} \frac{u_{2} r_{x x}}{r}+\beta \frac{u_{2} q_{x}}{r}, \\
u_{2 t}= & -\frac{\beta}{2} u_{1 x x}-2 \beta u_{2 x} q-\beta u_{2} q_{x}+2 \beta u_{1}-2 \beta u_{2} r+\frac{\beta}{2} \frac{u_{1} r_{x x}}{r}+\beta \frac{u_{1} q_{x}}{r}, \\
s_{1 t}= & -\frac{\beta}{2} s_{2 x x}-2 \beta s_{1 x} q-\beta s_{1} q_{x}+2 \beta s_{1} r-2 \beta s_{2} r^{2}-3 \beta s_{2}^{2} r+\beta s_{1}^{2} r+2 \beta s_{1} s_{2} \\
& \quad-\beta s_{2}^{3}+\beta s_{1}^{2} s_{2}+\frac{\beta}{2} \frac{s_{2} r_{x x}}{r}+\beta \frac{s_{2} q_{x}}{r}, \\
s_{2 t}= & -\frac{\beta}{2} s_{1 x x}-2 \beta s_{2 x} q-\beta s_{2} q_{x}+2 \beta s_{1}-2 \beta s_{2} r+3 \beta s_{1}^{2} r-\beta s_{2}^{2}-2 \beta s_{1} s_{2} r+\beta s_{1}^{3} \\
& \quad-\beta s_{1} s_{2}^{2}+\frac{\beta}{2} \frac{s_{1} r_{x x}}{r}+\beta \frac{s_{1} q_{x}}{r} .
\end{aligned}\right.
$$

Finally, we obtain the Hamiltonian structure of one of coupling integrable couplings of the generalized coupled Burgers equation hierarchy by using the quadratic-form identity as follows:

$$
u_{t}=J \frac{\delta H_{n+2}}{\delta u}=J L^{n}\left(\begin{array}{c}
0 \\
-\beta r-\beta u_{2}-\beta s_{2} \\
\beta \\
-\beta r \\
\beta+\beta s_{1} \\
-\beta r-\beta s_{2}
\end{array}\right) \text {. }
$$

In this paper we can only get the Hamiltonian structure of one of the coupling integrable couplings of the generalized coupled Burgers equation hierarchy by using the quadraticform identity. How to get all the Hamiltonian structure of one coupling integrable couplings of the generalized coupled Burgers equation hierarchy is worthy of further studying.

\section{Methods and experiment}

\section{Not applicable.}

\section{Acknowledgements}

The authors are grateful to the anonymous referees and the editor for their careful corrections and valuable comments on the original version of this paper.

\section{Funding}

The authors were supported by National Natural Science Foundation of China (Grant Nos. 11701320, 11705122), the Science and Technology Foundations of Shandong Province (Grant Nos. J16li52 and J14li54) and Science Foundations of Binzhou University (Grant Nos. BZXYL1104 and BZXYL1704).

\section{Competing interests}

The authors declare that they have no competing interests.

\section{Authors' contributions}

All authors contributed equally to the manuscript and read and approved the final manuscript.

\section{Author details}

${ }^{1}$ College of Science, Binzhou University, Binzhou City, P.R. China. ${ }^{2}$ Department of Applied Mathematics, Changsha University of Science and Technology, Changsha, P.R. China. ${ }^{3}$ Hunan Provincial Key Laboratory of Mathematical Modeling and Analysis in Engineering, Changsha, P.R. China.

\section{Publisher's Note}

Springer Nature remains neutral with regard to jurisdictional claims in published maps and institutional affiliations. 


\section{References}

1. Zhang, T.G.: The trace identity, a powerful tool for constructing the Hamiltonian structure of integrable systems. J. Math. Phys. 30(2), 330-339 (1989)

2. Wadati, M.: The modified Korteweg-de Vries equation. J. Phys. Soc. Jpn. 34, 1289-1296 (1973)

3. Gu, C., et al.: Soliton Theory and Its Application. Zhenjiang Publishing House of Science and Technology (1990)

4. Ma, W.X.: Integrable couplings of vector AKNS soliton equations. J. Math. Phys. 46, 033507 (2005)

5. Ma, W.X., Fuchssteiner, B.: The bi-Hamiltonian structure of the perturbation equations of the KdV hierarchy. Phys. Lett. A $213,49-55$ (1996)

6. Ma, W.X., Fuchssteiner, B.: Integrable theory of the perturbation equations. Chaos Solitons Fractals 7, 1227-1250 (1996)

7. Guo, F.K., Zhang, Y.F.: A new loop algebra and a corresponding integrable hierarchy as well, as its integrable coupling. J. Math. Phys. 44, 5793-5803 (2003)

8. Zhang, Y.F.: A generalized multi-component Glachette-Johnson (GJ) hierarchy and its integrable coupling system. Chaos Solitons Fractals 21(2), 305-310 (2004)

9. Cheng, X.T., Yu, F.J., Chen, D.Y.: The multi-component generalized Wadati-Konono-Ichikawa (WKI) hierarchy and its multi-component integrable couplings system with two arbitrary functions. Chaos Solitons Fractals 24, 877-883 (2005)

10. Guo, F.K.: Subalgebras of the loop algebra $\tilde{A_{1}}$ and integrable Hamiltonian hierarchies of equations. Acta Math. Phys. Sin. 19(5), 507-512 (1999)

11. Zhang, Y.F., Zhang, H.Q.: Integrable coupling of Td hierarchy. J. Math. Phys. 43(1), 466 (2002)

12. Zhang, Y.F: An integrable Hamiltonian hierarchy and its constrained flows with generalized Hamiltonian regular representations, as well as its expanding integrable system. Chaos Solitons Fractals 18(4), 855-862 (2003)

13. Cheng, X.T., Yu, F.J.: Multi-component Dirac equation hierarchy and its multi-component integrable couplings system. Chin. Phys. 16(3), 605-610 (2007)

14. Ma, W.X.: A new hierarchy of Liouville integrable generalized Hamiltonian equations and its reduction. Chin J. Contemp. Math. 13(1), 79 (1992)

15. Ma, W.X., Gao, L.: Coupling integrable couplings. Phys. Lett. B 23(15), 1847-1860 (2009)

16. Ma, W.X., Xu, X.X., Zhang, Y.F.: Semidirect sums of Lie algebras and discrete integrable couplings. J. Math. Phys. 47, $053501(2006)$

17. Mao, A.-M., Chen, Y.-S.: Existence and concentration of solutions for sublinear Schrödinger-Poisson equations. Indian J. Pure Appl. Math. 49(2), 339-348 (2018)

18. Zheng, X.-X., Shang, Y.-D., Di, F.-H.: The time-periodic solutions to the modified Zakharov equations with a quantum correction. Mediterr. J. Math. 14, 152 (2017)

19. He, X.M., Qian, A.X., Zou, W.M.: Existence and concentration of positive solutions for quasi-linear Schrödinger equations with critical growth. Nonlinearity 26,3137-3168 (2013)

20. Qian, A.X.: Infinitely many sign-changing solutions for a Schrödinger equation. Adv. Differ. Equ. 2011, 39 (2011)

21. Sun, Y., Liu, L.S., Wu, Y.H.: The existence and uniqueness of positive monotone solutions for a class of nonlinear Schrodinger equations on infinite domains. J. Comput. Appl. Math. 321, 478-486 (2017)

22. Yang, X.J., Gao, P., Srivastava, H.M.: A new computational approach for solving nonlinear local fractional PDEs. J. Comput. Appl. Math. 339, 285-296 (2018)

23. Yang, X.J., Gao, P., Srivastava, H.M.: Exact travelling wave solutions for the local fractional two-dimensional Burgers-type equations. Comput. Math. Appl. 73(2), 203-210 (2017)

24. Yang, X.J., Machado, J.T., Baleanu, D.: Exact traveling-wave solution for local fractional Boussinesq equation in fractal domain. Fractals 25(4), 1740006 (2017)

25. Guo, Y.X.: Exponential stability analysis of traveling waves solutions for nonlinear delayed cellular neural networks. Dyn. Syst. 32(4), 490-503 (2017)

26. Gao, P., Yang, X.J., Zhang, Y.F.: Exact traveling wave solutions for a new non-linear heat transfer equation. Therm. Sci. 21(4), 1833-1838 (2017)

27. Gao, P., Yang, X.J., Machado, T., Baleanu, D.: Exact Travelling Wave Solutions for Local Fractional Partial Differential Equations in Mathematical Physics. Mathematical Methods in Engineering, vol. 175. Springer, Cham (2018)

28. Tian, H.H., Han, M.A.: Bifurcation of periodic orbits by perturbing high-dimensional piecewise smooth integrable systems. J. Differ. Equ. 263, 7448-7474 (2017)

29. Sun, W.W.: Stabilization analysis of time-delay Hamiltonian systems in the presence of saturation. Appl. Math Comput. 217(23), 9625-9634 (2011)

30. Sun, W.W., Wang, Y.Z.: L2 disturbance attenuation for a class of time delay Hamiltonian systems. J. Syst. Sci. Complex 24(4), 672-682 (2011)

31. Wang, B., Yang, H., Meng, F.: Sixth order symplectic and symmetric explicit ERKN schemes for solving multi frequency oscillatory nonlinear Hamiltonian equations. Calcolo 54, 117-140 (2017)

32. Yusuf, A., Inc, M., Aliyu, A.l., Baleanu, D.: Conservation laws, soliton-like and stability analysis for the time fractional dispersive long-wave equation. Adv. Differ. Equ. 2018, 319 (2018)

33. Abdel-Gawad, H.I., Tantawy, M., Inc, M., Yusuf, A.: On multi-fusion solitons induced by inelastic collision for quasi-periodic propagation with nonlinear refractive index and stability analysis. Mod. Phys. Lett. B 32(29), 1850353 (2018)

34. Tchier, F., Inc, M., Yusuf, A., Aliyu, A.I., Baleanu, D.: Time fractional third-order variant Boussinesq system: symmetry analysis, explicit solutions, conservation laws and numerical approximations. Eur. Phys. J. Plus 133, 240 (2018)

35. Inc, M., Yusuf, A., Aliyu, A.I., Baleanu, D.: Lie symmetry analysis and explicit solutions for the time fractional generalized Burgers-Huxley equation. Opt. Quantum Electron. 50, 94 (2018)

36. Baleanu, D. Inc, M. Yusuf, A., Aliyu, A.l.: Lie symmetry analysis and conservation laws for the time fractional simplified modified Kawahara equation. Open Phys. 16, 302-310 (2018)

37. Inc, M., Yusuf, A., Aliyu, A.I., Baleanu, D.: Optimal system, nonlinear self-adjointness and conservation laws for generalized shallow water wave equation. Open Phys. 16, 364-370 (2018) 
38. Zhang, Y.F., Honwah, T.: Three kinds of coupling integrable couplings of the Korteweg-de Vries hierarchy of evolution equations. J. Math. Phys. 51, 043510 (2010)

39. Guo, F.Q., Zhang, Y.F.: The quadratic-form identity for constructing the Hamiltonian structure of integrable systems. J. Phys. A 38, 8537-8548 (2005)

Submit your manuscript to a SpringerOpen ${ }^{\odot}$ journal and benefit from:

- Convenient online submission

$\checkmark$ Rigorous peer review

- Open access: articles freely available online

- High visibility within the field

- Retaining the copyright to your article

Submit your next manuscript at $\gg$ springeropen.com 